\title{
ACAROLOGY
}

\section{Biologia de Amblyseius largoensis (Muma) (Acari: Phytoseiidae), um Potencial Predador de Aceria guerreronis Keifer (Acari: Eriophyidae) em Coqueiro}

\author{
Andréia S. Galvão ${ }^{1}$, Manoel G.C. Gondim Jr. ${ }^{1}$, Gilberto J. de Moraes² e José V. de Oliveira ${ }^{1}$ \\ ${ }^{1}$ Depto. Agronomia/Fitossanidade, Univ. Federal Rural de Pernambuco, Av. Dom Manoel de Medeiros, s/n \\ Dois irmãos, 52171-900, Recife, PE \\ ${ }^{2}$ Depto. Entomologia, Fitopatologia e Zoologia Agricola/Escola Superior de Agricultura “Luiz de Queiroz"/USP \\ Av. Pádua Dias, 11, 13418-900, Piracicaba, SP
}

Neotropical Entomology 36(3):465-470 (2007)

Biology of Amblyseius largoensis (Muma) (Acari: Phytoseiidae), a Potential Predator of Aceria guerreronis Keifer (Acari: Eriophyidae) on Coconut Trees

\begin{abstract}
Aceria guerreronis Keifer is considered one of the main pests of coconuts around the world. Amongst the Phytoseiidae recorded on this crop, Amblyseius largoensis (Muma) has been reported in association with A. guerreronis. In order to verify whether A. largoensis feeds on A. guerreronis, the biology of this predator was evaluated on different food sources, including A. guerreronis. Three types of diet were tested [Tetranychus urticae Koch + castor bean (Ricinus communis L.) pollen + honey at $10 \%$, A. guerreronis + pollen + honey, and only A. guerreronis], determining its development, survivorship, oviposition, sex ratio and longevity at $27^{\circ} \mathrm{C}, 60 \pm 5 \% \mathrm{RH} 12 \mathrm{~h}$ photophase. Fertility life tables were constructed. The duration of the immature phase was lower when feeding only on A. guerreronis, while fecundity was higher when feeding on a prey + pollen + honey. There was no difference in relation to survivorship of the immature stages between the three diets. Parameters of fertility life tables were higher when the diet included A. guerreronis or T. urticae + pollen + honey, although the predator was able to complete its life cycle and reproduce when feeding exclusively on $A$. guerreronis. The results suggest that A. largoensis preys upon A. guerreronis under natural condition and that it might play some role in the control of the latter.
\end{abstract}

KEY WORDS: Mite, biological control, Cocos nucifera

RESUMO - Aceria guerreronis Keifer é considerada uma das principais pragas do coqueiro no mundo. Dentre os Phytoseiidae relatados nessa cultura, Amblyseius largoensis (Muma) tem sido encontrado associado a $A$. guerreronis. Com o intuito de verificar se $A$. largoensis se alimenta de $A$. guerreronis, foi estudada a biologia desse predador com diferentes fontes de alimento, incluindo A. guerreronis. Foram avaliadas três dietas [Tetranychus urticae Koch + pólen de mamona (Ricinus communis L.) + mel a $10 \%$, A guerreronis + pólen + mel e A. guerreronis isoladamente], avaliando-se o desenvolvimento, sobrevivência, oviposição, razão sexual e longevidade de $A$. largoensis a $27^{\circ} \mathrm{C}, 60 \pm 5 \%$ de umidade relativa e fotofase de $12 \mathrm{~h}$. Tabelas de vida de fertilidade foram construídas. A duração da fase imatura foi menor na dieta constituída apenas por A. guerreronis, enquanto que a fecundidade foi maior nas dietas à base de presa + pólen + mel. Não houve diferença entre os valores de sobrevivência das formas imaturas submetidas a diferentes dietas alimentares. Os parâmetros da tabela de vida de fertilidade foram superiores quando a alimentação foi constituída por A. guerreronis ou T. urticae + pólen + mel, embora o predador tenha sido capaz de completar seu ciclo biológico e se reproduzir quando alimentado exclusivamente com A. guerreronis. Os resultados sugerem que A. largoensis seja um predador de A. guerreronis em condições naturais e que possa estar desempenhando algum papel no controle dessa praga.

PALAVRAS-CHAVE: Ácaro, controle biológico, Cocos nucifera 
Aceria guerreronis Keifer é considerada uma das principais pragas do coqueiro, (Cocos nucifera L.) na América, África e Ásia (Moore \& Howard 1996, Haq et al. 2002). As colônias desse eriofídeo localizam-se na região meristemática do fruto, coberta pelas brácteas (Alencar et al. 1999), onde causam danos freqüentemente muito significativos. Eventualmente podem atacar plantas jovens, provocando lesões que se expandem por toda folha, causando a morte da planta (Aquino et al. 1968). Em virtude dos danos, A. guerreronis provoca perdas significativas em todas as regiões produtoras do mundo, causando perda de peso, redução no tamanho e, conseqüentemente, no valor comercial dos frutos (Moore et al. 1989, Haq et al. 2002).

Os ácaros da família Phytoseiidae são considerados alguns dos agentes de controle biológico mais eficientes de ácaros fitófagos (McMurtry \& Croft 1997), principalmente devido ao baixo requerimento alimentar, rápido desenvolvimento, alta habilidade de forrageamento, persistência em plantas com baixa infestação de presas e pela capacidade de sobrevivência em substratos alternativos (Moraes 2002). Alguns trabalhos demonstraram que espécies de fitoseídeos podem ser importantes predadores de eriofídeos (Moraes \& Lima 1983, Sabelis \& Bruin 1996, Momen \& Hussein 1999) sendo alguns deles promissores na regulação de populações de A. guerreronis (Moraes \& Zacarias 2002). Seu comportamento fototrópico negativo pode, teoricamente, facilitar o encontro com aquela praga, que vive em lugares protegidos.

Diversas espécies de Phytoseiidae, dentre as quais Amblyseius largoensis (Muma), têm sido relatadas em coqueiro (Moraes et al. 2004). Não existe nenhum estudo sobre o papel desse predador como agente de controle de A. guerreronis. O objetivo deste trabalho foi verificar se $A$. largoensis utiliza $A$. guerreronis como fonte de alimento, baseando-se no ciclo biológico e nos parâmetros reprodutivos e, assim, avaliar se $A$. largoensis pode ser considerado um agente potencial de controle dessa praga.

\section{Material e Métodos}

O presente trabalho foi realizado no Laboratório de Acarologia Agrícola, da Área de Fitossanidade do Departamento de Agronomia da Universidade Federal Rural de Pernambuco (UFRPE).

Coleta, estabelecimento e criação de $\boldsymbol{A}$. largoensis. Os ácaros utilizados neste trabalho foram obtidos de coqueiros (C. nucifera) anões verdes, em fevereiro de 2005 do banco de germoplasma da UFRPE ( $8^{\circ} 01^{\prime} 02^{\prime}$ 'S e $\left.34^{\circ} 56^{\prime} 41^{\prime \prime} \mathrm{W}\right)$. Os ácaros foram criados no laboratório a $27,5 \pm 1,0^{\circ} \mathrm{C}, 75$ $\pm 10 \%$ U.R. e $12 \mathrm{~h}$ de fotofase. A unidade de criação foi constituída por um disco de folha cotiledonar de feijão-deporco (Canavalia ensiformes L.) de $13 \mathrm{~cm}$ de diâmetro, com a superfície abaxial voltada para cima, sobreposto a um disco de papel de filtro com $15 \mathrm{~cm}$ de diâmetro, colocado sobre um disco de espuma de polietileno de igual diâmetro e com $1 \mathrm{~cm}$ de espessura. Cada disco foi colocado no interior de uma bandeja plástica ( $16 \mathrm{~cm}$ de diâmetro) à qual se adicionou água destilada diariamente para manter a espuma umedecida, mantendo-se a folha úmida e evitando-se a fuga dos ácaros. Foram estabelecidas três colônias, cada uma alimentada com uma dieta diferente: 1. Tetranychus urticae Koch (Acari: Tetranychidae) + pólen de mamona (Ricinus communis L.) + mel a $10 \% ; 2$. A. guerreronis + pólen de mamona + mel a $10 \% ; 3$. A. guerreronis. O eriofídeo foi oferecido em pedaços da epiderme do fruto de aproximadamente $0,4 \times 0,4$ $\mathrm{cm}$, contendo em média 80 a 100 ácaros de todas as fases de desenvolvimento. Para a dieta contendo T. urticae foram oferecidas 15 fêmeas. O mel foi oferecido umedecendo-se um pedaço de papel toalha de $0,8 \times 0,8 \mathrm{~cm}$ colocado sobre uma lamínula de $1,8 \times 1,8 \mathrm{~cm}$, sendo o pólen também oferecido sobre uma lamínula de mesmo tamanho. Os alimentos foram adicionados diariamente.

Biologia de $A$. largoensis com diferentes fontes de alimento. Cerca de 400 fêmeas adultas de cada colônia foram transferidas para quatro unidades de estudo (100 fêmeas/unidade), semelhantes às anteriormente descritas. As unidades foram mantidas por $8 \mathrm{~h}$ a $27^{\circ} \mathrm{C}, 60 \pm 5 \%$ de umidade relativa e sob luz constante, com as mesmas dietas utilizadas na manutenção das colônias. Após esse período, as fêmeas foram retiradas e os ovos postos pelos predadores foram mantidos nas mesmas unidades, sob as mesmas condições de temperatura e umidade, e sob fotofase de $12 \mathrm{~h}$. A cada $6 \mathrm{~h}$, as unidades foram examinadas para determinar o período de incubação de cada ovo. Logo após a eclosão, cada larva foi transferida para uma nova unidade experimental, estabelecida de maneira semelhante ao que foi anteriormente descrito, mas com dimensão de $5 \times 5 \mathrm{~cm}$. A dieta em cada unidade foi reposta diariamente. A cada $6 \mathrm{~h}$, as unidades foram examinadas para determinar a viabilidade e duração de cada estágio imaturo. As unidades contendo machos adultos foram examinadas a cada $24 \mathrm{~h}$, para determinar a longevidade. Logo após a emergência de cada fêmea adulta, transferiu-se um macho da colônia de manutenção para a unidade em que esta se encontrava; machos mortos eram repostos durante o período de observação das fêmeas. Estas unidades também foram examinadas a cada $24 \mathrm{~h}$ para a determinação dos períodos de pré-oviposição, oviposição, pós-oviposição, longevidade e oviposição diária. Os ovos obtidos foram isolados para determinar a razão sexual.

O delineamento experimental foi inteiramente casualizado, com três tratamentos, cada um correspondendo a um tipo de alimentação das colônias, sendo 65,100 e 67 repetições, para a dieta 1 (T. urticae + pólen + mel a $10 \%)$, dieta $2(A$. guerreronis + pólen + mel a 10\% ) e dieta 3 (A. guerreronis), respectivamente. Os resultados foram submetidos à análise de variância, sendo as médias comparadas pelo Teste de Tukey $(\mathrm{P}=0,05)$, utilizando o programa computacional SANEST 3.0 (Zonta et al. 1986).

Tabela de vida de fertilidade de A. largoensis: Os parâmetros da tabela de vida de fertilidade de $A$. largoensis [taxa líquida de reprodução $\left(\mathrm{R}_{\mathrm{o}}\right)$, tempo médio da geração (T), taxa intrínseca de crescimento populacional $\left(\mathrm{r}_{\mathrm{m}}\right)$, taxa finita de crescimento populacional $(\lambda)$ e tempo médio em dias para duplicar a população em números (TD)] foram 
estimados através do programa computacional SAS (SAS Institute 1999-2001), adaptando o modelo escrito por Maia et al. (2000), o qual utiliza o método "Jackknife" para estimar intervalos de confiança das médias dos tratamentos e permite comparações entre pares de tratamentos empregando-se o teste "T".

\section{Resultados e Discussão}

Biologia de $A$. largoensis com diferentes fontes de alimento. $\mathrm{O}$ desenvolvimento de $A$. largoensis apresentou maior duração nos estágios de ovo $\left(F_{2,229}=13,25 ; \mathrm{P}<0,001\right)$, $\operatorname{larva}\left(F_{2,229}=120,54 ; \mathrm{P}<0,001\right)$ e protoninfa $\left(F_{2,229}=106,01\right.$; $\mathrm{P}<0,001)$ quando alimentado com a dieta 1 , entretanto, a duração do estágio deutoninfal foi similar nos tratamentos contendo a dieta 1 e $2\left(F_{2,229}=99,88 ; \mathrm{P}<0,001\right)$. A duração total de ovo a adulto diferiu entre as três dietas $\left(F_{2,229}=68,51\right.$; $\mathrm{P}<0,001)$, sendo maior para $A$. largoensis alimentado com a dieta 1 e menor quando alimentado com a dieta 3. Em relação à sobrevivência, não houve diferença significativa entre as dietas para cada estágio imaturo $(86,6 \%$ a $100 \%)$ e no período de ovo a adulto $(75,3 \%$ a $84,2 \%)$ (Tabela 1$)$.

Kamburov (1971) constatou a influencia de várias fontes de alimento no desenvolvimento e na fecundidade de $A$. largoensis. O menor tempo de desenvolvimento foi registrado nos tratamentos compostos isoladamente por tetraniquídeos, tenuipalpídeos e pólen, ou com a adição de secreções açucaradas. Segundo o autor, A. largoensis alimentado com Phyllocoptruta oleivora (Ashmead) (Acari: Eriophyidae) + secreções açucaradas atingiu a fase adulta em 5,5 dias, mas não completou o ciclo biológico quando alimentado exclusivamente com o eriofídeo. Já no presente trabalho, o menor tempo de desenvolvimento foi obtido com a dieta 3 , também composta por um eriofídeo.
A. largoensis alimentado com a dieta 1 apresentou menor período de pré-oviposição $\left(F_{2,107}=15,99 ; \mathrm{P}<0,001\right)$. O período de oviposição foi significativamente maior nas dietas 1 e $2\left(F_{2,107}=148,63 ; \mathrm{P}<0,001\right)$, enquanto que o período de pós-oviposição foi significativamente superior na dieta $1\left(F_{2,107}=14,50 ; \mathrm{P}<0,001\right)$. A longevidade de fêmeas e machos foi maior quando o predador foi alimentado com as dietas 1 e $2\left(F_{2,107}=87,88 ; \mathrm{P}<0,001\right.$ e $F_{2,107}=12,20 ; \mathrm{P}$ $<0,001$, respectivamente), quando comparada a dieta 3 . A oviposição total e diária foram significativamente superiores nas dietas 1 e $2\left(F_{2,107}=242,72 ; \mathrm{P}<0,001\right.$ e $F_{2,107}=36,44 ; \mathrm{P}$ $<0,001$, respectivamente). Não foram observadas diferenças significativas na razão sexual do predador entre as três dietas (Tabela 2). Nas três dietas, a taxa máxima de oviposição ocorreu logo no início da fase adulta, entre o quarto e o sexto dia, observando-se após esse período uma queda lenta nesta taxa. A sobrevivência das fêmeas atingiu o nível de $50 \%$ aos 26, 24 e 8 dias nas dietas 1,2 e 3, respectivamente (Figs. 1 e 2).

A oviposição e o nível de sobrevivência de 50\% das fêmeas na dieta 3 foram também menores que o determinado por outros autores (Kamburov 1971, Yue \& Tsai 1996, Rodriguez \& Ramos 2004) para A. largoensis recebendo diferentes tipos de alimento.

Tabela de vida de fertilidade de $\boldsymbol{A}$. largoensis. Todos os parâmetros biológicos das tabelas de vida de fertilidade foram significativamente inferiores na dieta 3 , entretanto, esses valores não diferiram entre as dietas 1 e 2 (Tabela 3). Esses resultados corroboram aqueles citados anteriormente neste trabalho (Tabela 2), especialmente no que se refere aos níveis de oviposição que foram significativamente maiores nas dietas 1 e 2 , quando comparados com a dieta 3 .

Os resultados obtidos nas dietas 1 e 2 são compatíveis com aqueles obtidos por outros autores para $A$. largoensis

Tabela 1. Duração média em dias ( \pm EP) e sobrevivência (\%) dos estágios imaturos de A. largoensis em três dietas alimentares. Temp. $27^{\circ} \mathrm{C}$, UR $60 \pm 5 \%$ e fotofase de $12 \mathrm{~h}$.

\begin{tabular}{|c|c|c|c|c|}
\hline \multirow[b]{2}{*}{ Fase } & \multicolumn{4}{|c|}{ Dietas } \\
\hline & & $\begin{array}{c}1 \\
\text { T. urticae }+ \text { pólen }+ \text { mel } \\
(\mathrm{n}=65)\end{array}$ & $\begin{array}{c}2 \\
\text { A. guerreronis }+ \text { pólen }+ \text { mel } \\
(\mathrm{n}=100)\end{array}$ & $\begin{array}{c}3 \\
\text { A. guerreronis } \\
(\mathrm{n}=67)\end{array}$ \\
\hline \multirow{2}{*}{ Ovo } & Duração & $1,7 \pm 0,01 \mathrm{a}$ & $1,4 \pm 0,01 \mathrm{~b}$ & $1,4 \pm 0,02 b$ \\
\hline & Sobrevivência & $100,0 \pm 0,00 \mathrm{a}$ & $100,0 \pm 0,00 \mathrm{a}$ & $100,0 \pm 0,00 \mathrm{a}$ \\
\hline \multirow{2}{*}{ Larva } & Duração & $1,0 \pm 0,01 \mathrm{a}$ & $0,8 \pm 0,01 \mathrm{~b}$ & $0,8 \pm 0,01 \mathrm{~b}$ \\
\hline & Sobrevivência & $90,6 \pm 3,38 \mathrm{a}$ & $92,5 \pm 2,28 \mathrm{a}$ & $96,6 \pm 2,46 \mathrm{a}$ \\
\hline \multirow{2}{*}{ Protoninfa } & Duração & $1,1 \pm 0,02 \mathrm{a}$ & $0,8 \pm 0,01 \mathrm{c}$ & $0,9 \pm 0,01 \mathrm{~b}$ \\
\hline & Sobrevivência & $95,1 \pm 2,39 \mathrm{a}$ & $96,3 \pm 1,82$ a & $88,1 \pm 3,50 \mathrm{a}$ \\
\hline \multirow{2}{*}{ Deutoninfa } & Duração & $1,2 \pm 0,02 \mathrm{a}$ & $1,2 \pm 0,02 \mathrm{a}$ & $0,9 \pm 0,02 b$ \\
\hline & Sobrevivência & $87,5 \pm 3,75 \mathrm{a}$ & $93,5 \pm 2,24 \mathrm{a}$ & $86,6 \pm 3,90 \mathrm{a}$ \\
\hline \multirow{2}{*}{ Ovo-adulto } & Duração & $5,0 \pm 0,03 \mathrm{a}$ & $4,2 \pm 0,03 b$ & $4,0 \pm 0,01 \mathrm{c}$ \\
\hline & Sobrevivência & $77,6 \pm 4,58$ a & $84,2 \pm 3,35 \mathrm{a}$ & $75,3 \pm 4,16$ a \\
\hline
\end{tabular}

Médias seguidas da mesma letra na linha não diferem entre si, pelo teste de Tukey $(\mathrm{P}=0,05)$. 
Tabela 2. Duração média ( \pm EP), em dias, dos períodos de pré-oviposição, oviposição e pós-oviposição, oviposição diária e total, longevidade e razão sexual de A. largoensis em três dietas alimentares. Temp. $27{ }^{\circ} \mathrm{C}$, UR $60 \pm 5 \%$ e fotofase de $12 \mathrm{~h}$.

\begin{tabular}{|c|c|c|c|}
\hline \multirow{4}{*}{ Parâmetros biológicos } & \multicolumn{3}{|c|}{ Dietas } \\
\hline & 1 & 2 & 3 \\
\hline & T. urticae + pólen + mel & A. guerreronis + pólen + mel & A. guerreronis \\
\hline & $\mathrm{n}_{\uparrow}=37 ; \mathrm{n}_{0}=17$ & $\mathrm{n}_{+}=40 ; \mathrm{n}_{0}=14$ & $\mathrm{n}_{+}=33 ; \mathrm{n}_{0}=11$ \\
\hline Período de pré-oviposição & $0,9 \pm 0,01 \mathrm{c}$ & $1,3 \pm 3,84 \mathrm{~b}$ & $1,7 \pm 0,13 \mathrm{a}$ \\
\hline Período de oviposição & $20,4 \pm 0,58 \mathrm{a}$ & $20,0 \pm 0,79 \mathrm{a}$ & $5,3 \pm 0,56 b$ \\
\hline Período de pós-oviposição & $5,3 \pm 0,63 \mathrm{a}$ & $1,9 \pm 0,25 \mathrm{~b}$ & $2,9 \pm 0,43 b$ \\
\hline Oviposição diária & $1,7 \pm 0,04 \mathrm{a}$ & $1,7 \pm 0,04 \mathrm{a}$ & $1,2 \pm 0,08 \mathrm{~b}$ \\
\hline Oviposição total & $33,2 \pm 1,07 \mathrm{a}$ & $33,9 \pm 0,86 \mathrm{a}$ & $6,4 \pm 0,77 \mathrm{~b}$ \\
\hline Longevidade fêmea & $26,6 \pm 0,84 \mathrm{a}$ & $23,3 \pm 1,26 \mathrm{a}$ & $9,8 \pm 0,60 \mathrm{~b}$ \\
\hline Longevidade macho & $15,6 \pm 1,00 \mathrm{a}$ & $14,0 \pm 0,76 \mathrm{a}$ & $4,7 \pm 0,52 b$ \\
\hline Razão sexual & $0,71 \pm 0,01 \mathrm{a}$ & $0,71 \pm 0,01 \mathrm{a}$ & $0,69 \pm 0,01 \mathrm{a}$ \\
\hline
\end{tabular}

Médias seguidas da mesma letra na linha não diferem entre si, pelo teste de Tukey $(\mathrm{P}=0,05)$.

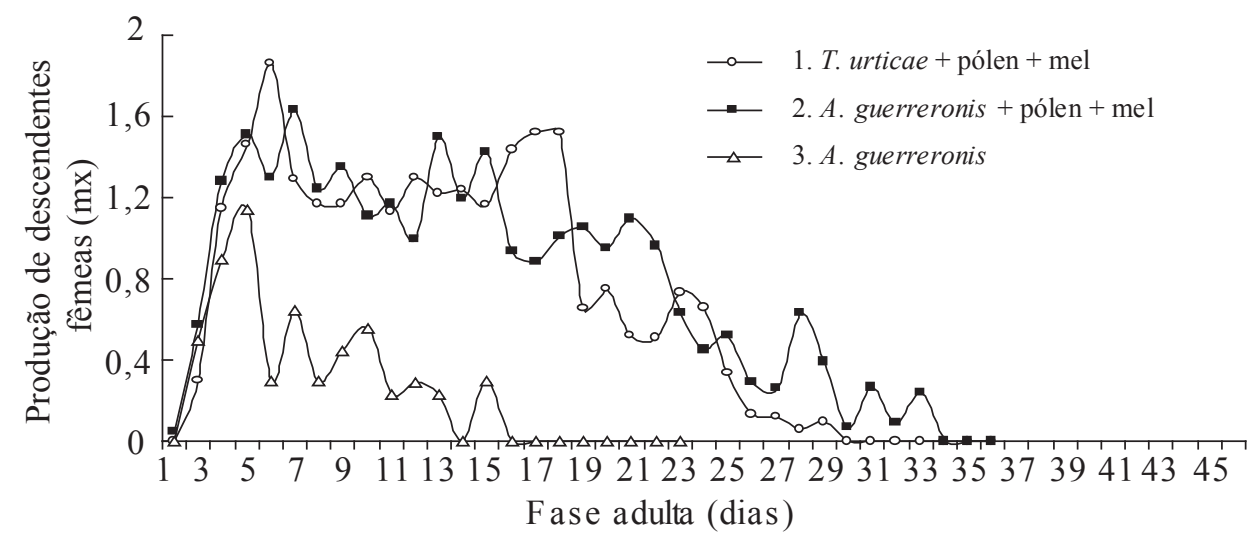

Fig. 1. Ritmo de produção de descendentes fêmeas (mx) de A. largoensis, nas três dietas alimentares. Temp. $27^{\circ} \mathrm{C}, \mathrm{UR} 60 \pm$ $5 \%$ e fotofase de $12 \mathrm{~h}$.

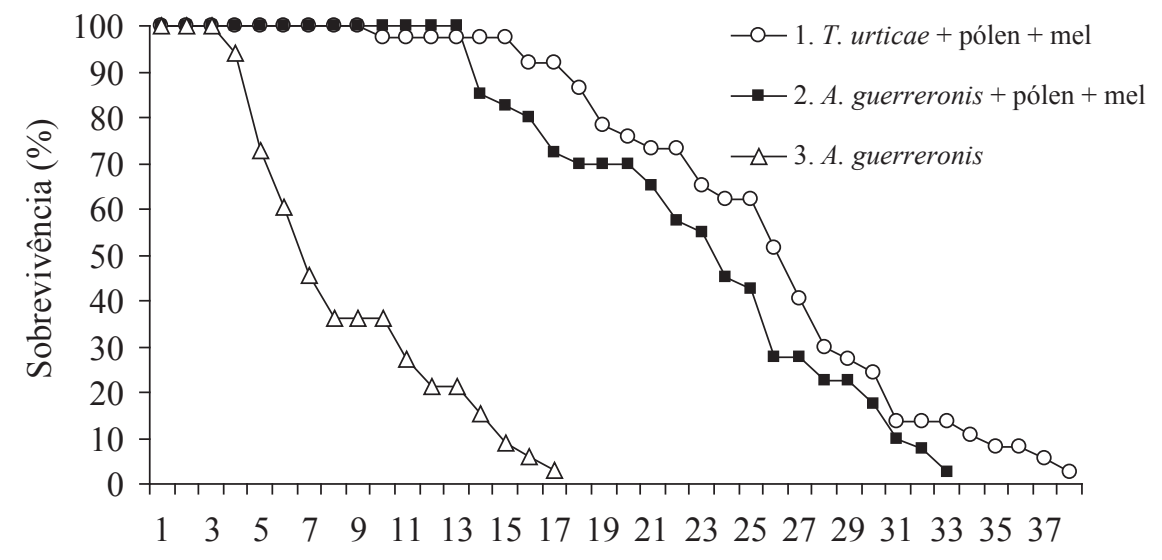

Fase adulta (dias)

Fig. 2. Sobrevivência (\%) de fêmeas de A. largoensis, nas três dietas alimentares. Temp. $27^{\circ} \mathrm{C}$, UR $60 \pm 5 \%$ e fotofase de $12 \mathrm{~h}$. 
Tabela 3. Parâmetros da tabela de vida de fertilidade registrados para A. largoensis em três dietas alimentares. $\left(\mathrm{R}_{\mathrm{o}}\right)$ Taxa líquida de reprodução, (T) tempo médio de geração, $\left(\mathrm{r}_{\mathrm{m}}\right)$ taxa intrínseca de crescimento populacional, $(\lambda)$ taxa finita de crescimento populacional e (TD) tempo médio em dias para duplicar a população em números. Temp. $27^{\circ} \mathrm{C}$, UR $60 \pm$ $5 \%$ e fotofase de $12 \mathrm{~h}$.

\begin{tabular}{lccccc}
\hline Dietas & $\begin{array}{c}\mathrm{R}_{\mathrm{o}} \\
(+)(+)^{-1}\end{array}$ & $\begin{array}{c}\mathrm{T} \\
(\mathrm{dias})\end{array}$ & $\begin{array}{c}\mathrm{r}_{\mathrm{m}} \\
(+)(+)^{-1}(\mathrm{dia})^{-1}\end{array}$ & $\lambda$ & $\begin{array}{c}\text { TD } \\
(\text { dias })\end{array}$ \\
\hline 1. T. urticae + pólen + mel & 19,42 & 12,21 & 0,24 & 1,27 & 2,85 \\
& $(18,33-20,50) \mathrm{a}$ & $(11,81-12,61) \mathrm{a}$ & $(0,23-0,24) \mathrm{a}$ & $(1,26-1,28) \mathrm{a}$ & $(2,77-2,93) \mathrm{a}$ \\
2. A. guerreronis + pólen + mel & 20,75 & 12,24 & 0,25 & 1,28 & 2,80 \\
& $(19,12-22,37) \mathrm{a}$ & $(11,78-12,70) \mathrm{a}$ & $(0,24-0,25) \mathrm{a}$ & $(1,27-1,29) \mathrm{a}$ & $(2,71-2,88) \mathrm{a}$ \\
3. A. guerreronis & 3,41 & 6,79 & 0,18 & 1,20 & 3,80 \\
& $(2,75-4,07) \mathrm{b}$ & $(6,20-7,38) \mathrm{b}$ & $(0,15-0,20) \mathrm{b}$ & $(1,16-1,22) \mathrm{b}$ & $(3,28-4,32) \mathrm{b}$ \\
\hline
\end{tabular}

Médias seguidas pela mesma letra na coluna, não diferem entre si por meio de comparações de tratamentos dois a dois através do intervalo de confiança a $95 \%$ de probabilidade após estimativa de erros pelo método Jackknife (SAS Institute 1999-2001).

recebendo outros tipos de alimento por eles considerados como favoráveis (Yue \& Tsai 1996, Morell \& Lima 2003).

Os resultados obtidos neste trabalho indicam que $A$. largoensis preda $A$. guerreronis, contudo este não pode ser considerado um alimento muito adequado para o predador quando oferecido isoladamente, como indicado pela baixa oviposição e longevidade verificada na dieta 3. Entretanto, é interessante observar que mesmo na dieta 3 , a razão intrínseca de crescimento populacional foi relativamente elevada, quando os dados são comparados aos obtidos para outros fitoseídeos. McMurtry \& Croft (1997) consideram A. largoensis como generalista do tipo III, que pode se aproveitar de vários tipos diferentes de alimento, incluindo diferentes espécies de eriofídeos, alguns pequenos insetos, pólen, substâncias açucaradas, etc. No habitat ocupado por A. largoensis sobre os coqueiros, as fontes de alimento são diversificadas, podendo o predador encontrar pólen, secreções açucaradas de insetos sugadores, o que beneficia seu desenvolvimento e reprodução.

Além da possível limitação fisiológica, sugerida pelos resultados dos parâmetros avaliados na presença exclusiva de $A$. guerreronis como alimento, há ainda a limitação física ao desempenho de A. largoensis como agente de controle da praga, relacionada ao grande tamanho desse predador em relação às dimensões do habitat ocupado pela praga, sob as brácteas, o que freqüentemente dificulta seu acesso às suas colônias. No entanto, há que se considerar que na fase de migração, A. guerreronis ocupa a superfície de diferentes órgãos vegetais (Moore \& Alexander 1987), expondo-se e podendo então ser atacados por esse e por outros predadores. Assim, é de se esperar que nessas ocasiões $A$. largoensis possa contribuir, juntamente com outros fitoseídeos, para a redução da população da praga.

\section{Agradecimentos}

À CAPES e CNPq pela concessão da bolsa de estudo junto ao Programa de Pós-Graduação em Fitossanidade/ Entomologia da UFRPE.

\section{Referências}

Alencar, J.A., F.N.P. Haji \& F.R.B. Moreira. 1999. Ácaro-da-necrosedo-coqueiro Aceria guerreronis: Aspectos bioecológicos, sintomas, danos e medidas de controle. Petrolina, Embrapa Semi-árido, 18p. (Embrapa Semi-arido, Documento).

Aquino, M.L.N., C.H.W. Flechtmann \& G.P. Arruda. 1968. Contribuição ao estudo do ácaro causador da necrose do olho do coqueiro em Pernambuco e seu controle. Recife, IPA, 17p. (Boletim Técnico, 34).

Haq, M.A., K. Sumangala \& N. Ramani. 2002. Coconut mite invasion, injury and distribution, p.41-49. In L.C.P. Fernando, G.J. Moraes \& I.R. Wickramananda (eds.), Proceedings of the International Workshop on Coconut Mite (Aceria guerreronis). Sri Lanka, Coconut Research Institute, 117p.

Kamburov, S.S. 1971. Development and reproduction of Amblyseius largoensis on various food substances. J. Econ. Entomol. 64: 643-648.

Maia, A.H.N., A.J.B. Luiz \& C. Campanhola. 2000. Statistical inference on associated fertility life table parameters using Jackknife technique: Computational aspects. J. Econ. Entomol. 93: 511-518.

McMurtry, J.A. \& B.A. Croft. 1997. Life styles of phytoseiid mites and their roles in biological control. Annu. Rev. Entomol. 42: 291-321.

Momen, F.M. \& H. Hussein. 1999. Relationships between food substances, developmental success and reproduction in Typhlodromus transvaalensis (Acari: Phytoseiidae). Acarologia 40: 108-111.

Moore, D. \& F.W. Howard. 1996. Coconuts, p.561-570. In E.E. Lindquist, M.W. Sabelis \& J. Bruin (eds.), Eriophyoid mites: Their biology, natural enemies and control. Amsterdam, Elsevier, 790p.

Moore, D. \& L. Alexander. 1987. Aspects of migration and colonization of the coconut palm by the coconut mite, Eriophyes guerreronis (Keifer) (Acari: Eriophyidae). Bull. Entomol. Res. 77: 641-650. 
Moore, D., L. Alexander \& R.A. Hall. 1989. The coconut mite, Eriophyes guerreronis Keifer in St Lucia yield losses and attempts to control it with acaricide, polybutene e Hirsutella fungus. Trop. Pest Manag. 35: 83-89.

Moraes, G.J. 2002. Controle biológico de ácaros fitófagos com predadores, p.225-237. In J.R.P. Parra, P.S.M. Botelho, B.S. Corrêa-Ferreira \& J.M.S. Bento (eds.), Controle biológico: Parasitóides e predadores. São Paulo, Manole, 635p.

Moraes, G.J. \& H.C. Lima. 1983. Biology of Euseius concordis (Chant) (Acari: Phytoseiidae) a predator of the tomato russet mite. Acarologia 24: 251-255.

Moraes, G.J., J.A. McMurtry, H.A. Denmark \& C.B. Campos. 2004. A revised catalog of the mite family Phytoseiidae. Zootaxa 434: $1-494$.

Moraes, G.J. \& M.S. Zacarias. 2002. Use of predatory mites for control of eriophyid mites, p.78-88. In L.C.P. Fernando, G.J. Moraes \& I.R. Wickramananda (eds.), Proceedings of the International Workshop on Coconut Mite (Aceria guerreronis). Sri Lanka, Coconut Research Institute, 117p.

Morell, H.R. \& M.R. Lima. 2003. Evaluación de métodos de cria del ácaro Amblyseius largoensis. Manejo Integr. Plagas Agroecol. 70: 55-64.
Rodriguez, H. \& M. Ramos. 2004. Biology and feeding behavior of Amblyseius largoensis (Muma) Acari: Phytoseiidae) on Polyphagotarsonemus latus (Banks) (Acari: Tarsonemidae). Rev. Proteccion Veg. 19: 73-79.

SAS Institute. 1999-2001. SAS/STAT User's guide, version 8.02, TS level 2MO. SAS Institute Inc., Cary, NC.

Sabelis, M.W. \& J. Bruin. 1996. Evolutionary ecology: Life history patterns, food plant choice and dispersal, p.329-366. In E.E. Lindquist, M.W. Sabelis \& J. Bruin (eds), Eriophyoid mites: Their biology, natural enemies and control. Amsterdam, Elsevier, 790p.

Yue. B. \& J.H. Tsai. 1996. Development, survival and reproduction of Amblyseius largoensis (Acari: Phytoseiidae) on selected plant pollens and temperatures. Environ. Entomol. 25: 487494.

Zonta, E.P., P. Silveira \& A.A. Machado. 1986. Sistema de análise estatística (SANEST 3.0). Pelotas, Instituto de Física e Matemática, UFPel, 399p.

Received 27/III/06. Accepted 10/II/07. 